\title{
Cosmogenic Activation of Materials Used in Rare Event Search Experiments
}

\author{
C. Zhang ${ }^{\text {a }}$ D.-M. Mei ${ }^{\mathrm{a}, \mathrm{b}, *}$ V. A. Kudryavtsev ${ }^{\mathrm{c}}$ S. Fiorucci $^{\mathrm{d}}$ \\ ${ }^{a}$ Department of Physics, The University of South Dakota, Vermillion, SD 57069 \\ ${ }^{\mathrm{b}}$ School of Physics and Optoelectronic, Yangtze University, Jingzhou 434023, \\ China \\ ${ }^{\mathrm{c}}$ Department of Physics and Astronomy, University od Sheffield, Sheffield S3 $7 R H$, \\ $U K$ \\ ${ }^{\mathrm{d}}$ Lawrence Berkeley National Laboratory, 1 Cyclotron Rd., Berkeley CA 94720, \\ $U S A$
}

\begin{abstract}
We evaluate the cosmogenic production rates in some materials that are commonly used as targets and shielding/supporting components for detecting rare events. The results from Geant4 simulations and the calculations of ACTIVIA are compared with the available experimental data. We demonstrate that the production rates from the Geant4-based simulations agree with the available data reasonably well. As a result, we report that the cosmogenic production of several isotopes in various materials can generate potential backgrounds for direct detection of dark matter and neutrinoless double-beta decay.
\end{abstract}

Key words: Cosmogenic activation, Dark matter detection, Geant4 Simulation PACS: 13.85.Tp, 23.40-s, 25.40.Sc, 28.41.Qb, 95.35.+d, 29.40.Wk

\section{Introduction}

Evidence from galactic and extragalactic observations indicate the existence of dark matter in our universe [1-3]. As a favored dark matter candidate, the Weakly Interacting Massive Particle (WIMP) can be directly detected by underground experiments through its elastic scattering off ordinary target

* Corresponding author.

Email address: Dongming.Mei@usd.edu (D.-M. Mei). 
materials $[4,5]$. None of the current dark matter experiments has convincingly observed WIMP scattering events. The next generation ton-scale dark matter experiments, especially the xenon-based detectors [6,7] are designed to achieve ultra-low background conditions thus allowing detection sensitivity to WIMP-nucleon spin-independent cross section down to $\sim 10^{-48} \mathrm{~cm}^{2}$. In order to achieve such a sensitivity level, the background rate in the region of interest needs to be at a maximum level of $\sim 0.1-0.2$ events/ton-year [6].

Neutrinoless double-beta decay experiments are aimed at measuring the effective Majorana mass of the electron neutrino down to 10 to $50 \mathrm{meV}$ to understand the nature (Dirac or Majorana) of neutrinos [8-11]. This requires measurements of a half-life for a nucleus at a level of $>10^{27}$ years. Existing experiments have achieved [12-14] or will soon achieve [15] a sensitivity of the order of $10^{25}$ years for several isotopes and set an upper limit on the effective Majorana mass of electron neutrino $<0.2 \mathrm{eV}$. Eventually, these future experiments $[12,13,15-17]$ target a sensitivity of $>10^{27}$ y or $<1$ event/ton-year in the region of interest to explore mass values favoured by both inverted and normal mass ordering.

Such low-background event rates for both dark matter and neutrinoless doublebeta decay require the radioactivity level of every detector component to be accurately measured. In addition to natural radioactivity, cosmogenic activation can add more radioactivity to a detector component. Mitigating measures such as underground storage as early as possible can be employed, but accurate activation calculations are needed to make decisions on design and operations. The activation of materials for underground experiments has been evaluated using ACTIVIA [18,19], TALYS [20], and other tools [12,15]. The discrepancy in the estimated activation rates between different tools exists and this deserves an investigation of the sources that may cause the discrepancy.

In this paper, we evaluate cosmogenic production of radioactive isotopes at sea level in various materials used for rare event experiments. The activation rates are obtained using Geant4-based simulations [21] and the calculations of ACTIVIA [22]. The results are compared with some experimental data $[18,19]$.

\section{Evaluation of cosmogenic production of radioactive isotopes on the surface}

\subsection{Evaluation tools and input energy spectra}

The Geant4 (V9.5p02)+Shielding modular physics list [24] is used for this study. It includes a set of electromagnetic and hadronic physics processes, 
with modular physics, boson physics, lepton physics, hadron physics, meson physics, nucleon physics, hyperon physics, antibaryon physics, ion physics, and Quark Gluon string model $(>20 \mathrm{GeV})$, Fritiof string model $(>5 \mathrm{GeV})$, Bertini-style Cascade $(<10 \mathrm{GeV})$, as well as high precision neutron model $(<20 \mathrm{MeV})$, required for high energy or underground detector simulations. For each physics model, G4MuonNuclearProcess was activated to simulate muon-nuclear inelastic scattering.

The modified Gaisser's formula [25,28] (Eq.(2) in Ref. [28]) is used to sample muons right above the simulation geometry. The energy range spans $1 \mathrm{GeV}$ to $100 \mathrm{TeV}$. The total flux is normalized to be $0.014 \mathrm{~cm}^{-2} \mathrm{~s}^{-1}$, which is the total muon flux corresponding to the energy range from $1 \mathrm{GeV}$ to $100 \mathrm{TeV}$ on the surface [25]. The stopping muons were not included in the simulation due to a much smaller flux [26,27]. Although there is a non-negligible fraction of stopping muons at the surface, the main contribution to the cosmogenic activation comes from atmospheric neutrons so stopping muons can be neglected.

For surface neutrons, in the Geant4 Monte Carlo simulation, the neutron spectrum from thermal neutrons to about $100 \mathrm{GeV}$ neutrons is used. The input energy spectrum of neutrons comes from the MCNPX simulation code [29] for energies below $4 \mathrm{MeV}$ and the New York data ("NY data") [30] for energies greater than $4 \mathrm{MeV}$ and is shown in Fig. 1. The neutron energy spectrum simulated by MCNPX code [29] is normalized to "NY data" for neutrons with energy, $E_{n}$, greater than $4 \mathrm{MeV}$ [30]. We use normalization to keep the entire spectrum continuous. This normalization allows us to calculate the total neutron flux and compare the result to the measured value [30,31]. Since cosmogenic activation of materials is mainly through neutron capture and neutron inelastic scattering processes and the latter requires a reaction threshold energy to be usually greater than $4 \mathrm{MeV}$, we report the output of the activation rates split by neutron energy:(1) thermal neutrons to fast neutrons with energy up to $4 \mathrm{MeV}$ and (2) fast neutrons with energy greater than $4 \mathrm{MeV}$. The energy spectrum for the range of $1 \times 10^{-8} \mathrm{MeV}$ (thermal neutrons) to 4 $\mathrm{MeV}$ (fast neutrons) adopts the results from MCNPX simulation [29] and its total flux is normalized to be $0.002 \mathrm{~cm}^{-2} \mathrm{~s}^{-1}$, which is the total neutron flux for this energy range $[29,30]$. For fast neutrons with energy greater than $4 \mathrm{MeV}$, the measurements of "NY data" [30] is used and its total flux is normalized to be $0.004 \mathrm{~cm}^{-2} \mathrm{~s}^{-1}$ [30]. It is worth mentioning that the $\mathrm{MeV}$ neutron data may have a small contamination from local radioactivity, which could slightly overestimate the neutron rate from cosmic rays in that range. Below the cosmogenic activation rates (atoms $\mathrm{kg}^{-1} \mathrm{day}^{-1}$ ) from the Geant4 simulation are reported according to the production mechanisms - muon spallation, neutron capture $\left(E_{n}<4 \mathrm{MeV}\right)$, and neutron inelastic scattering $\left(E_{n}>4 \mathrm{MeV}\right)$.

ACTIVIA [22] is a package that calculates cosmogenic production from cosmicray activation using data tables and semi-empirical formulas. ACTIVIA uses 


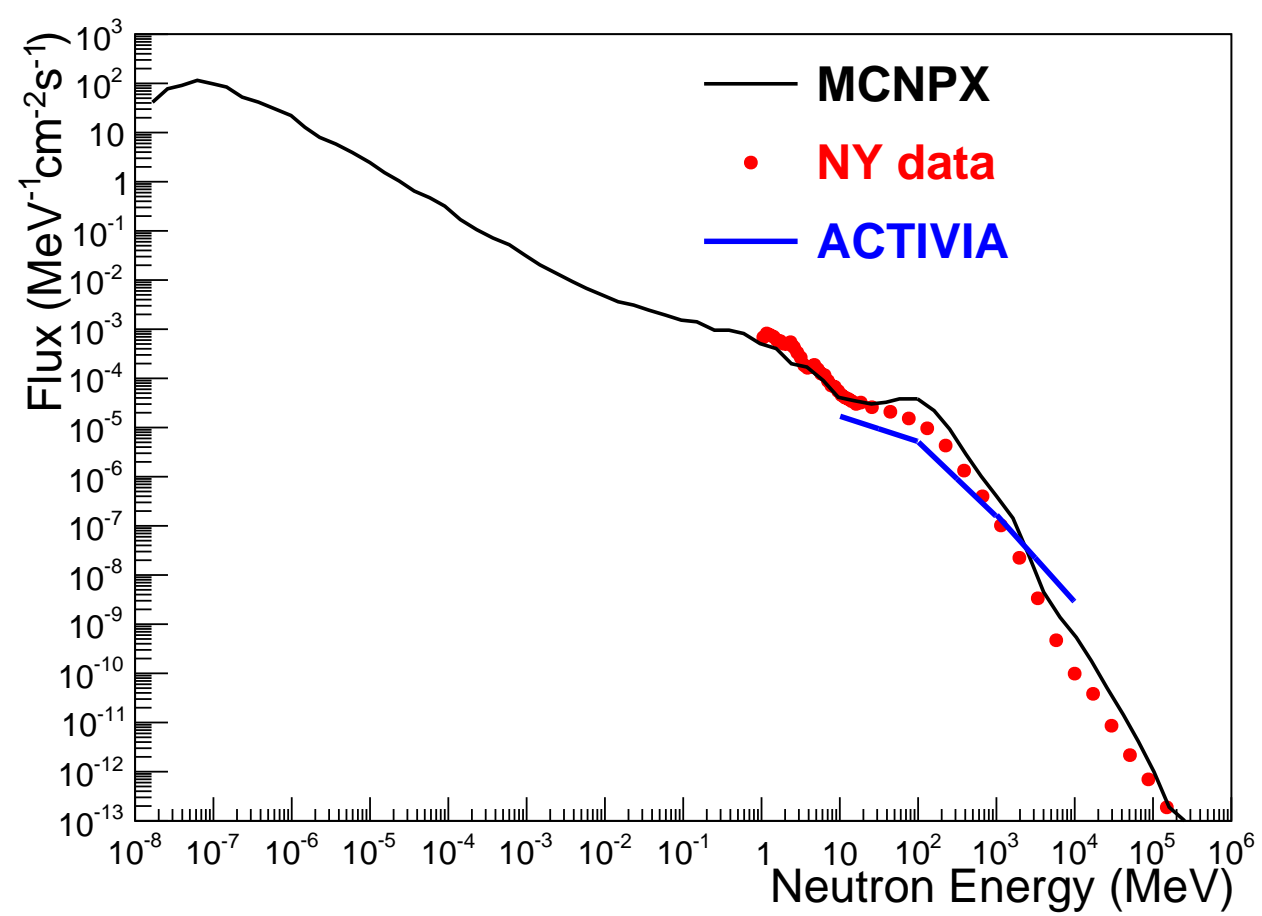

Fig. 1. The surface neutron energy spectra adopted in the evaluation. The black curve is the air shower simulation result using MCNPX package [29]. The red dots are the measured neutron spectrum at the surface [30]. The black curve is normalized to the data for $E_{n}>4 \mathrm{MeV}$. The blue line is a default input spectrum used by ACTIVIA package.

the neutron spectrum at the Earth surface shown by the blue curve in Fig. 1. Since this blue curve, the default neutron energy spectrum in ACTIVIA, is different from the input energy spectrum used in the Geant4 simulation, we also carried out ACTIVIA calculations using the neutron energy spectrum from the "NY" data as in the Geant4 simulation, in order to evaluate any difference between the two energy spectra. Therefore, "ACTIVIA1" stands for ACTIVIA calculation results with the blue line as the input neutron energy spectrum. "ACTIVIA2" represents ACTIVIA results with the red dots as its input neutron energy spectrum.

One of our goals is to compare ACTIVIA and Geant4 calculation of activation. In addition we compare ACTIVIA calculations of activation using two different neutron energy spectra. Since ACTIVIA is widely used in the field of low-background experiments for calculating cosmogenic activation, this study may be useful for future evaluation of activation of materials and estimating associated uncertainties. The overall difference in the neutron flux is about a factor of 3 between these two input fast neutron spectra.

Note that proton activation of materials is also included in the simulation 
although the number of protons below a few $\mathrm{GeV}$ is less than the number of neutrons in the atmosphere. The hadronic part of the cosmic-ray spectrum at the surface is dominated by neutrons [32]. Proton flux at low energies (below a few $\mathrm{GeV}$ ) is suppressed because of the proton energy losses. Proton spectrum is harder than the neutron one and protons dominate at energies exceeding a few $\mathrm{GeV}$. The activation rates of materials from protons, with energy spectrum from CRY [33], are provided in Table 1-5. It is worth mentioning that ACTIVIA does not include thermal neutron capture.

The variation of muon and neutron fluxes as a function of altitude can be described as [32]:

$$
I_{a}=I_{0} \exp \left(\frac{\left(A_{0}-A_{a}\right)}{\lambda}\right)
$$

where $I_{a}$ is the flux at a given altitude in meters, $I_{0}$ is the flux at the sea level, $A_{0}$ and $A_{a}$ are the atmospheric thickness in $\mathrm{g} / \mathrm{cm}^{2}$ for the sea level and any given altitudes, $\lambda$ is the average absorption length of particles in $\mathrm{g} / \mathrm{cm}^{2}$ in atmosphere. At the small altitudes, typical absorption lengths for muons and neutrons are $\lambda_{\mu}=520 \mathrm{~g} / \mathrm{cm}^{2}$ and $\lambda_{n}=148 \mathrm{~g} / \mathrm{cm}^{2}$ [32]. Fig. 2 shows relative intensities as a function of altitude. As an example, the altitude of the surface laboratory at the Sanford Underground Research Facility (SURF) is $\sim 1600$ meters above sea level [18]. At this altitude, the muon flux is a factor 1.41, and the neutron flux is a factor 3.34 higher than at sea level. To evaluate cosmogenic activation rates at different altitudes, the flux correction factors must be taken into account. The production rates are also dependent on the energy spectrum of muons and neutrons, but in practice the correction of the fluxes is the dominant effect. The variation in energy spectra of muons and neutrons is small for altitudes lower than 2000 meters above sea level. Although there are variations in cosmic radiation flux at different latitudes and longitudes, the main contribution to the cosmogenic activation comes from high-energy neutrons, which do not vary more than $1 \%$ in flux for different latitudes and longitudes [32].

\subsection{Cosmogenic production rates in xenon}

Liquid xenon is widely used as a target material in dark matter and neutrinoless double-beta decay experiments. It is generally sealed within gas cylinders made of stainless steel for storage and transportation purposes. Although natural xenon is stable, it suffers from cosmic-ray bombardment at the Earth surface. The cosmic rays produce radioactive xenon isotopes. The resulting activity can be estimated from the exposure time of xenon to cosmic rays on the surface since the time when xenon was produced. 


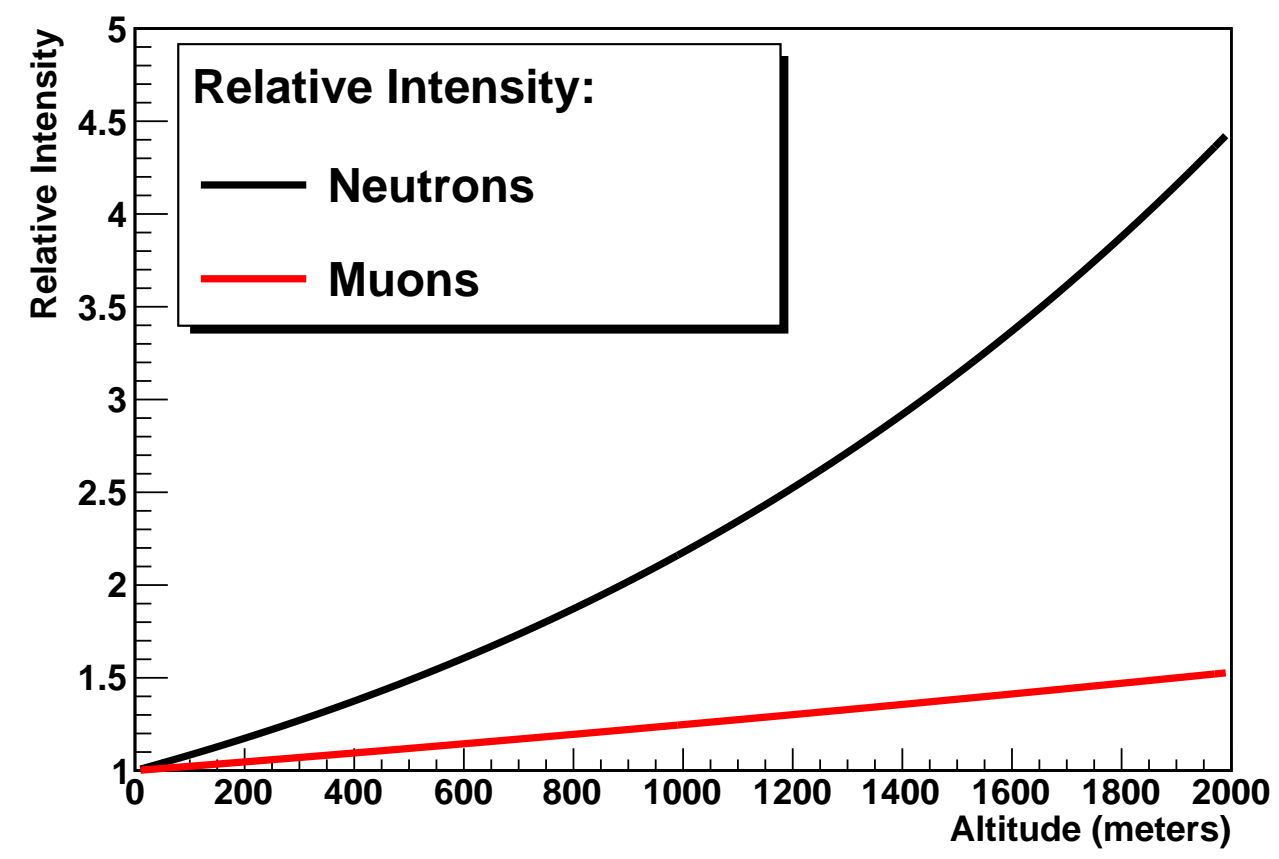

Fig. 2. The relative intensity of muons and neutrons as a function of altitude.

A comprehensive Geant4 (V9.5p02) [21] simulation has been conducted to evaluate the rate of xenon activation.

The xenon gas is assumed to be stored in a stainless steel cylinder that is 30.48 $\mathrm{cm}$ in diameter, $127 \mathrm{~cm}$ in height and $0.762 \mathrm{~cm}$ in thickness. The pressure of the gas is set to be 970 psi gauge with a density of $0.3657 \mathrm{~g} / \mathrm{cm}^{3}$ and temperature of $20{ }^{\circ} \mathrm{C}$. The total mass of the compressed gas is calculated to be $30 \mathrm{~kg}$ per cylinder. The cosmic-ray muons and neutrons are considered as inputs shot at a xenon gas cylinder. The cosmogenic production of xenon isotopes at the surface is dominated by neutron inelastic interactions.

Impurities other than radioactive xenon in xenon target can be eliminated by purification. However, radioactive xenon isotopes generated by cosmogenic activations cannot be removed during the purification. Low-energy neutrons, typically at thermal energies, activate target materials mainly through capture processes. The dangerous neutron capture in xenon targets are: ${ }^{126} \mathrm{Xe}(n, \gamma){ }^{127} \mathrm{Xe}$, ${ }^{132} \mathrm{Xe}(n, \gamma){ }^{133} \mathrm{Xe}$, and ${ }^{134} \mathrm{Xe}(n, \gamma){ }^{135} \mathrm{Xe}$.

In contrast, high-energy neutrons or muons can break stable xenon nuclei and convert them into radioactive isotopes, such as ${ }^{127} \mathrm{Xe},{ }^{133} \mathrm{Xe},{ }^{135} \mathrm{Xe},{ }^{125} \mathrm{I}$, ${ }^{129} \mathrm{I},{ }^{121} \mathrm{Te},{ }^{123} \mathrm{Te}$, etc. Among these production processes, neutron inelastic scattering reactions, ${ }^{128} \mathrm{Xe}(n, 2 n){ }^{127} \mathrm{Xe},{ }^{129} \mathrm{Xe}(n, 3 n){ }^{127} \mathrm{Xe},{ }^{134} \mathrm{Xe}(n, 2 n){ }^{133} \mathrm{Xe}$, ${ }^{136} \mathrm{Xe}(n, 2 n){ }^{135} \mathrm{Xe}$, dominate the production of radioactive xenon isotopes as shown in Table 1. 


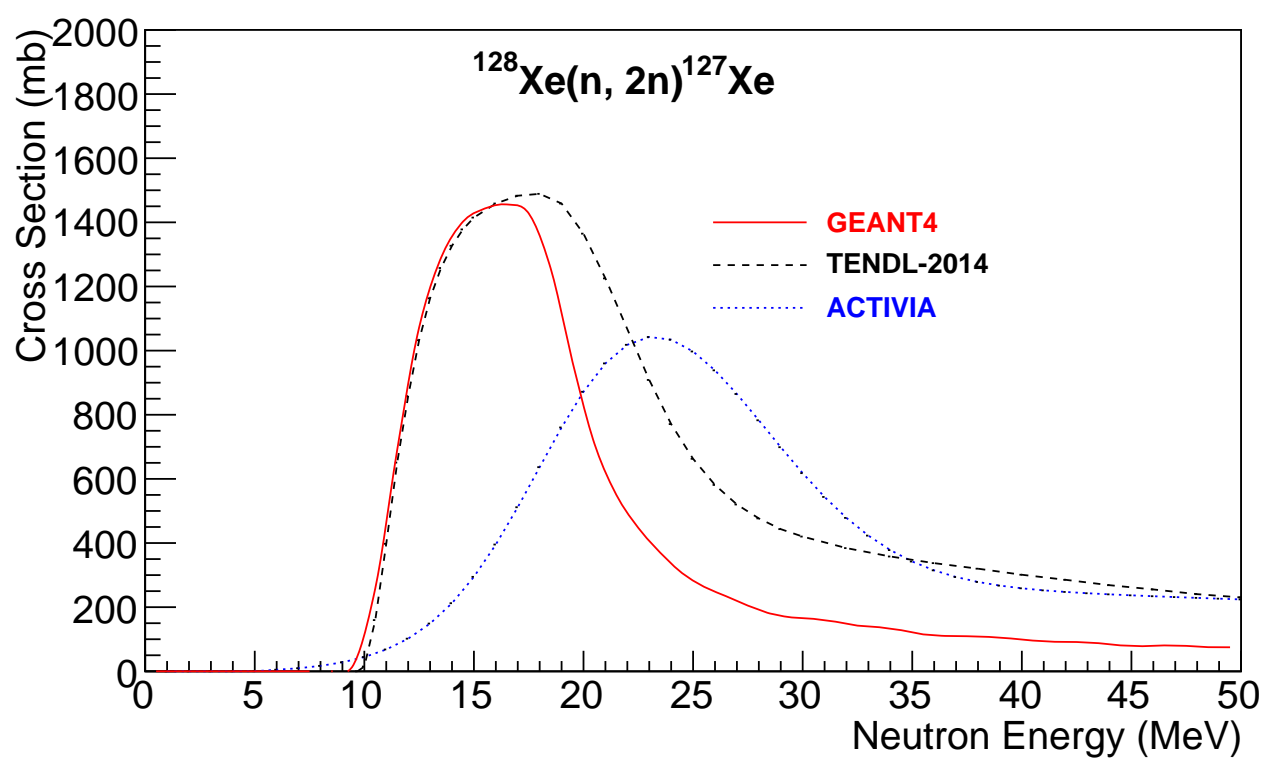

Fig. 3. Cross-sections comparison for the production of ${ }^{127} \mathrm{Xe}$ through ${ }^{128} \mathrm{Xe}(\mathrm{n}, 2 \mathrm{n}){ }^{127} \mathrm{Xe}$ between Geant4, ACTIVIA, and TENDL-2014 [34], which is a default cross-section library for TALYS-1.8 [34].

In the Geant4-based simulation, we track the initial and secondary particles to estimate the cosmogenic production rates. For a given input spectrum of muons and neutrons, using a Geant4-based simulation and an ACTIVIA package, the cosmogenically activated isotopes and their corresponding production rates are shown in Table 1. Results presented in columns 3 and 6 (the 2nd value) were obtained with the same neutron spectrum from "NY data" above $4 \mathrm{MeV}$. The difference in the production rates is then mainly due to the difference in the cross section libraries used in the Geant4-based simulation and ACTIVIA package and due to missing neutron transport and thermal neutron capture in ACTIVIA. Note that the difference in the production rates due to the lack of tracking capability, which takes into account the scattering/backscattering processes, in ACTIVIA is expected to be small since the simulated targets are small. We illustrate some differences in the cross sections for ${ }^{128} \mathrm{Xe}(n, 2 n){ }^{127} \mathrm{Xe},{ }^{129} \mathrm{Xe}(n, 3 n){ }^{127} \mathrm{Xe},{ }^{134} \mathrm{Xe}(n, 2 n){ }^{133} \mathrm{Xe}$ in Figures 3, 4, 5.

By default ACTIVIA uses the semi-empirical cross sections from Silberberg and Tsao $[35,36]$ but the possibility to use a different set of cross sections from MENDL libraries [40] is foreseen although these cross sections are not provided with ACTIVIA. We have not used MENDL libraries in this work.

TENDL is a nuclear data library used in the TALYS nuclear physics code. Geant4 uses a combination of the evaluated data libraries (ENDF/B-VII.0 [37], JEFF-3.1 [38], JENDL-4.0 [39], etc). As can be seen in Figures 3, 4, 5, the cross sections from Genat4 are similar to the cross sections from TENDL. The cross sections from ACTIVIA are significantly different from Genat4 and 


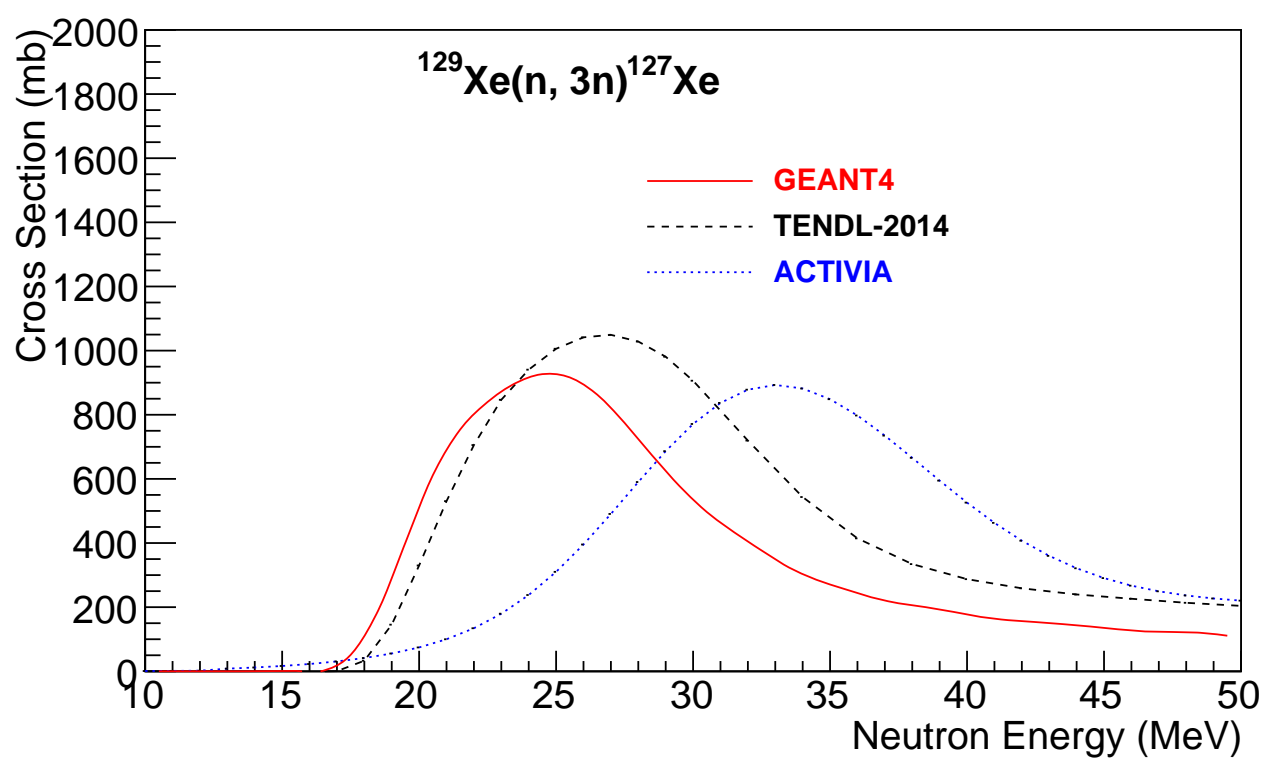

Fig. 4. Cross-sections comparison for the production of ${ }^{127} \mathrm{Xe}$ through ${ }^{129} \mathrm{Xe}(\mathrm{n}, 3 \mathrm{n}){ }^{127} \mathrm{Xe}$ between Geant4, ACTIVIA, and TENDL-2014.

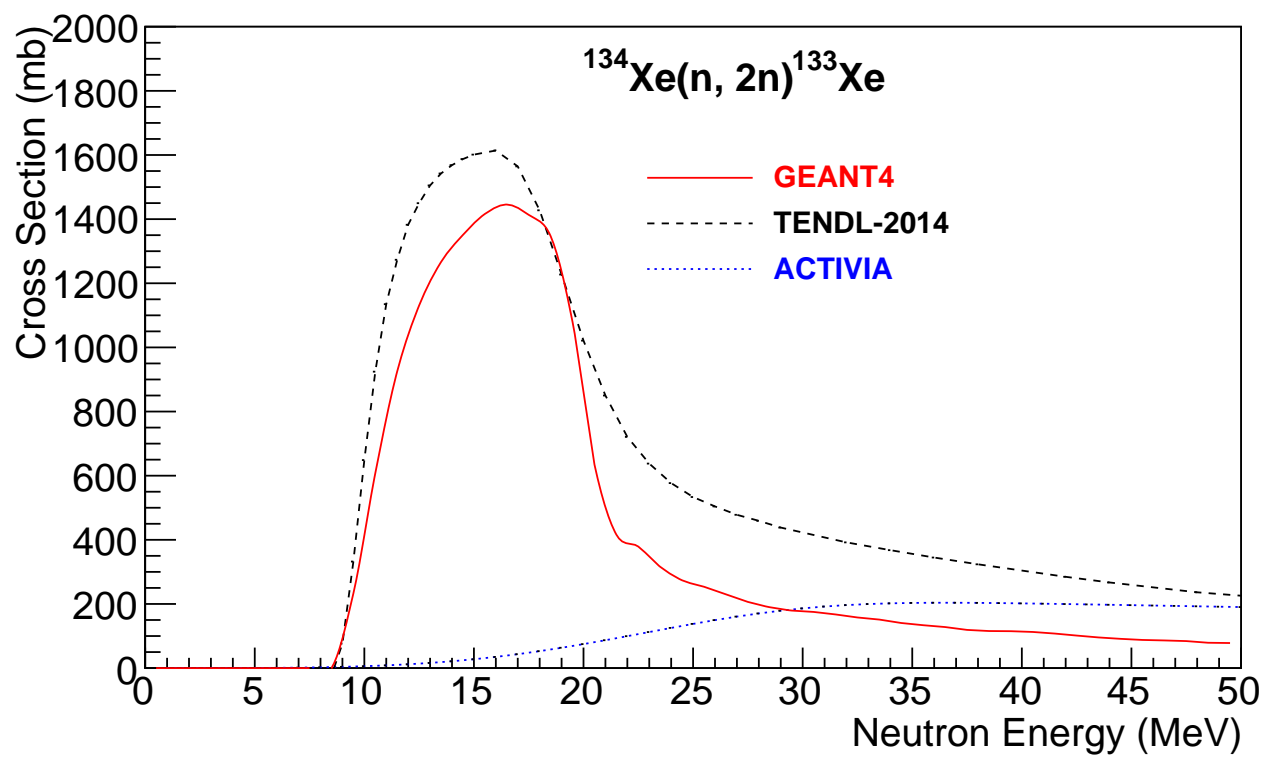

Fig. 5. Cross-sections comparison for the production of ${ }^{133} \mathrm{Xe}$ through ${ }^{134} \mathrm{Xe}(\mathrm{n}, 2 \mathrm{n}){ }^{133} \mathrm{Xe}$ between Geant4, ACTIVIA, and TENDL-2014.

TENDL. 
Table 1

Cosmogenic production rates in xenon gas at the sea level. Columns $2-5$ are obtained from the Geant 4 simulation.

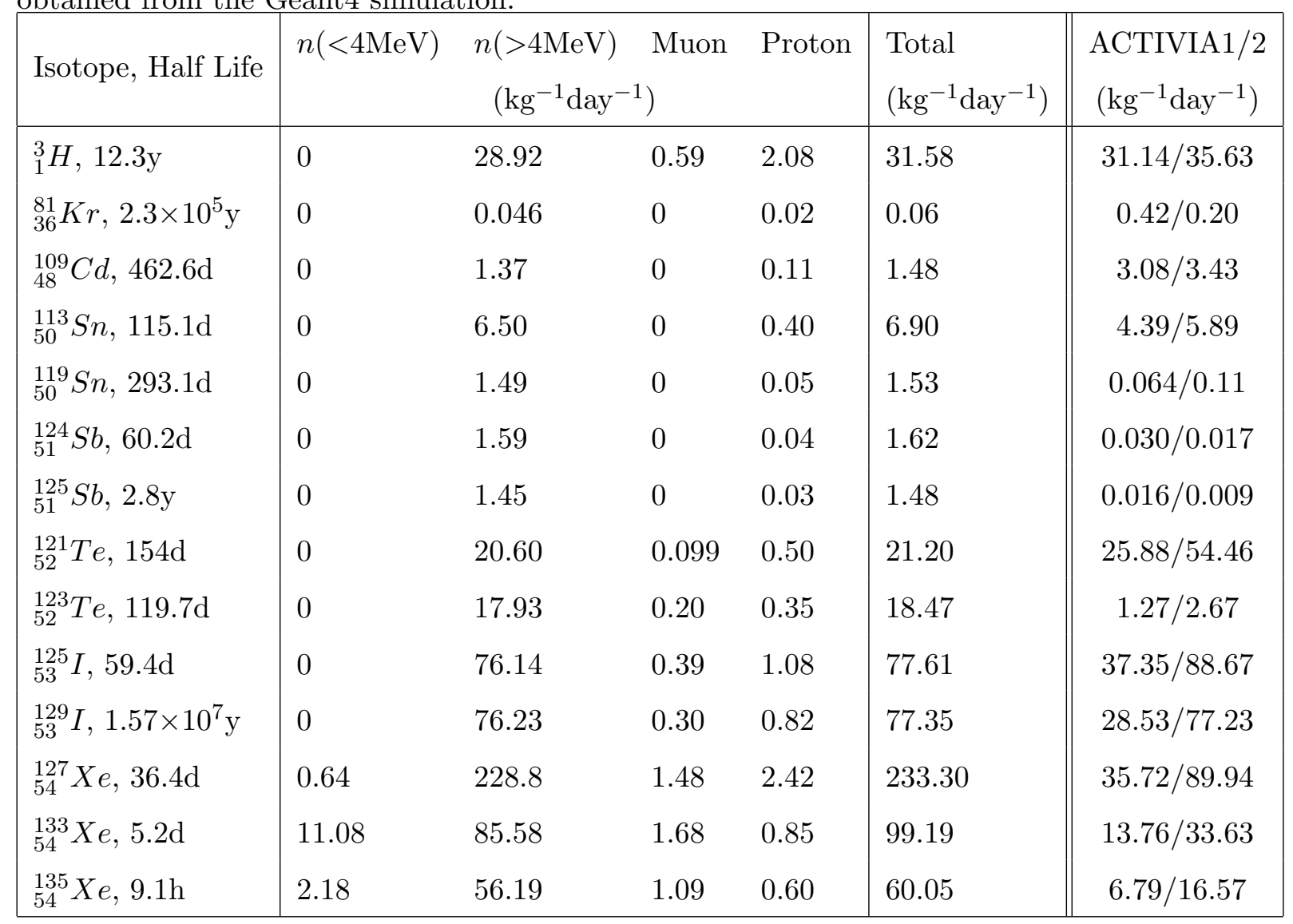

\subsection{Cosmogenic production rates in some key components of the rare event physics experiments}

Other than the xenon target itself, key materials, such as polytetrafluoroethylene (PTFE), copper, titanium, and stainless steel, which are generally used to build a detector, can also contribute to radioactivity. In the simulations, a bulk cylinder of these materials is assumed with $10 \mathrm{~cm}$ in diameter and $10 \mathrm{~cm}$ in height for evaluation.

PTFE is commonly used as an excellent reflector in experiments detecting light from scintillations, for instance in liquid xenon detectors. Cosmic rays can produce radioactive isotopes such as ${ }^{7} \mathrm{Be},{ }^{10} \mathrm{Be}$ and ${ }^{14} \mathrm{C}$ in PTFE. The simulated production rates are listed in Table 2 . The difference in the production rates between the Geant 4 simulation and ACTIVIA2 caused by cross sections ranges from a factor of 4 to 3000 . However, the production difference due to the difference in neutron flux between ACTIVIA1 and ACTIVIA2 is only about a factor of 2 . 
Table 2

Cosmogenic production in PTFE at the sea level. Columns $2-5$ give Geant4 results.

\begin{tabular}{|l|llll|l||c|}
\hline Isotope,Half Life & $\mathrm{n}(<4 \mathrm{MeV})$ & $\begin{array}{c}\mathrm{n}(>4 \mathrm{MeV}) \\
\left(\mathrm{kg}^{-1} \mathrm{day}^{-1}\right)\end{array}$ & Muon & Proton & $\begin{array}{l}\text { Total } \\
\left(\mathrm{kg}^{-1} \mathrm{day}^{-1}\right)\end{array}$ & $\begin{array}{l}\text { ACTIVIA1 } / 2 \\
\left(\mathrm{~kg}^{-1} \mathrm{day}^{-1}\right)\end{array}$ \\
\hline${ }_{4}^{7} B e, 53.1 \mathrm{~d}$ & 0.00 & 15.80 & 0.04 & 0.96 & 16.81 & $27.88 / 60.81$ \\
${ }_{4}^{10} \mathrm{Be}, 1.5 \times 10^{6} \mathrm{y}$ & 0.00 & 65.34 & 0.05 & 0.95 & 66.35 & $4.99 / 9.01$ \\
${ }_{6}^{14} \mathrm{C}, 5.7 \times 10^{3} \mathrm{y}$ & 0.09 & 0.01 & 0.00 & 0.00 & 0.10 & $13.74 / 29.62$ \\
\hline
\end{tabular}

Oxygen-free high-conductivity copper (OFHC) or electroformed copper can be made highly radiopure. The radiopure copper is commonly used as shielding and structural material for low-background detectors. The concern for this material when it is produced at the surface is the cosmogenically produced isotopes such as ${ }^{60} \mathrm{Co},{ }^{57} \mathrm{Co},{ }^{54} \mathrm{Mn}$. The cosmogenic production rates for copper are shown in Table 3. The difference in the production rates between the Geant4 simulation and ACTIVIA2 caused by cross sections ranges from a factor of $1.05\left({ }^{60} \mathrm{Co}\right)$ to $9\left({ }^{44} \mathrm{Ti}\right)$. A good agreement between the Geant4 simulation and ACTIVIA2 for the production of ${ }^{60} \mathrm{Co}$ shown in Table 3 is not due to the agreement in cross sections used in the two packages. In fact, the cross sections for the main production channel, ${ }^{63} \mathrm{Cu}(n, \alpha){ }^{60} \mathrm{Co}$, in Geant 4 and ACTIVIA are very different as can be seen in Figure 6. The difference between the cross-sections is then compensated by other effects such as the energy dependence of the cross-section and neutron flux, leading to an apparent similarity in production rates. Large cross-section in ACTIVIA is achieved at higher energies where the neutron flux is smaller, but extends to much higher neutron energies than the Geant 4 cross-section. Thus the convolution of the cross-section with the neutron flux done numerically (ACTIVIA) or by Monte Carlo (Geant4) may lead to very similar results.

The difference in the production rates due to the different fluxes between ACTIVIA1 and 2 is within a factor of 2 , but not very meaningful given the previous remark. Note that ${ }^{60} \mathrm{Co}$, produced through ${ }^{63} \mathrm{Cu}(n, \alpha){ }^{60} \mathrm{Co}$, is a main concern for both direct detection of dark matter and neutrinoless double-beta decay experiments because it has a long half-life (5.3 years) and emits two gamma rays $(1.173 \mathrm{MeV}$ and $1.333 \mathrm{MeV}$ ) with a summed energy up to 2.506 $\mathrm{MeV}$. These two gamma rays undergoing Compton scattering can generate background events in the region of interest for dark matter experiments. Two gamma rays can summed up to $2.506 \mathrm{MeV}$ energy, which is in the region of interest for neutrinoless double-beta decay experiments.

Stainless steel is normally used to build detector vessels, or as a structural material for low-background experiments [41,42]. Titanium was first adopted as a vessel material for liquid xenon by the LUX experiment [43] because a very radiopure sample has been found. The simulated cosmogenic production rates 


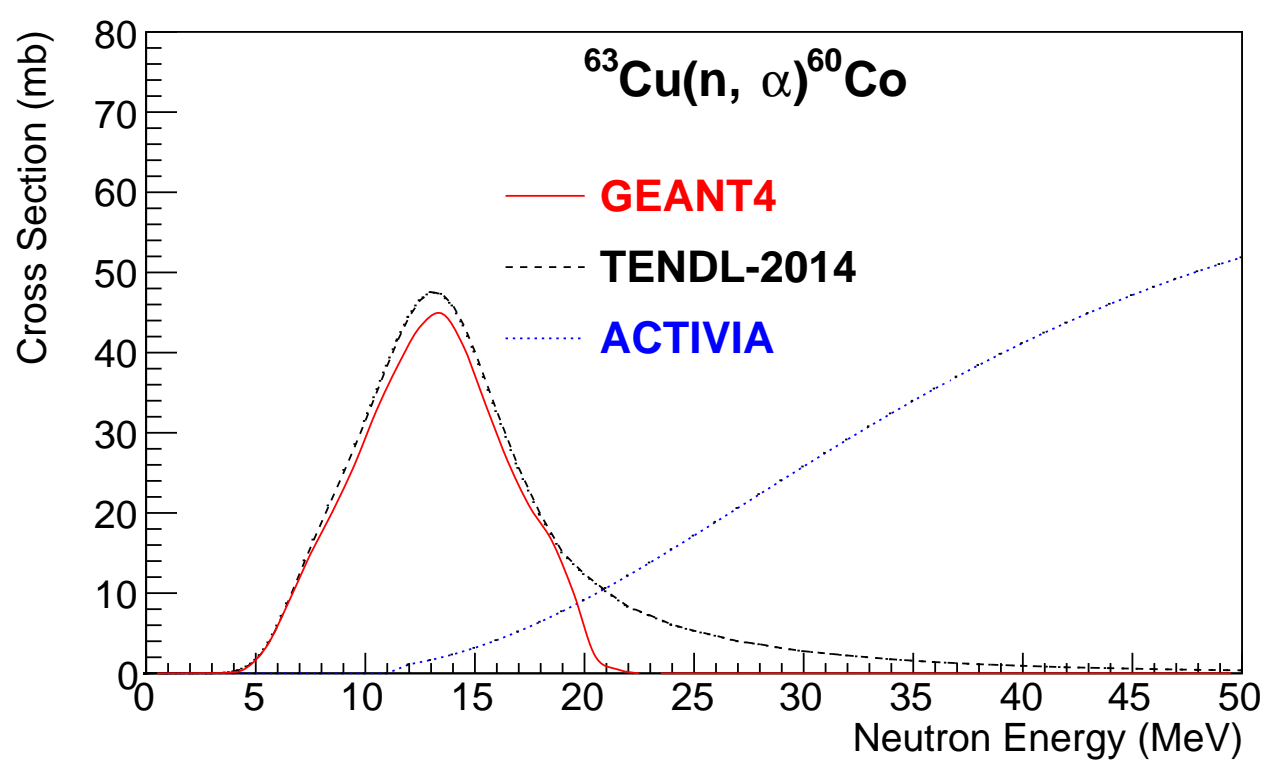

Fig. 6. The cross-section comparison for the production of ${ }^{60} \mathrm{Co}$ through ${ }^{63} \mathrm{Cu}(n, \alpha){ }^{60} \mathrm{Co}$ between Geant4, ACTIVIA, and TENDL-2014.

for stainless steel and titanium can be found in Table 4 and 5 . As can be seen in Table 4, there is a good agreement for the production rate of ${ }^{46} \mathrm{Sc}$ between the Geant4 simulation and ACTIVIA2 with the same neutron energy spectrum, although as can be seen in Figure 7, the cross sections are different by about a factor 2. A factor of about 2 caused by the cross section difference is seen in the production of ${ }^{46} \mathrm{Sc}$ in stainless steel between the Geant4 simulation and ACTIVIA2 (Table 5). Note that ${ }^{46} \mathrm{Sc}$ with a half-life of 83.8 days is a concern to dark matter experiments because it produces two simultaneous gamma rays with energies $0.889 \mathrm{MeV}$ and $1.121 \mathrm{MeV}$ that can undergo Compton scattering to generate background events in the region of interest. The production of ${ }^{46} \mathrm{Sc}$ in titanium is through ${ }^{46} \mathrm{Ti}(n, \mathrm{p})^{46} \mathrm{Sc}$ and in stainless steel is via spallation processes.

\section{Comparison between calculations and the available experimental data}

As can be seen from Tables 1-5, the results from the calculations using ACTIVIA (with default cross sections from $[35,36]$ ) are inconsistent with the simulated results from Geant4 for many isotopes. The inconsistencies can be caused by (1) the neutron energy spectrum and (2) the library for cross sections of inelastic scattering processes. To understand which simulation tool delivers more reliable results, we compared the Geant4 simulation results with AC- 
Table 3

Cosmogenic production in copper at the sea level. Columns $2-5$ give Geant4 results.

\begin{tabular}{|l|llll|l||c|}
\hline Isotope,Half Life & $\mathrm{n}(<4 \mathrm{MeV})$ & $\mathrm{n}(>4 \mathrm{MeV})$ & Muon & Proton & $\begin{array}{l}\text { Total } \\
\left(\mathrm{kg}^{-1} \mathrm{day}^{-1}\right)\end{array}$ & $\begin{array}{c}\text { ACTIVIA1 } / 2 \\
\left(\mathrm{~kg}^{-1} \mathrm{day}^{-1}\right)\end{array}$ \\
\hline${ }_{11}^{22} \mathrm{Na}, 2.6 \mathrm{y}$ & 0 & 0.012 & 0.0027 & 0.002 & 0.014 & $0.31 / 0.19$ \\
${ }_{13}^{26} \mathrm{Al}, 7.2 \times 10^{5} \mathrm{y}$ & 0 & 0.016 & 0.0027 & 0.002 & 0.021 & $0.23 / 0.14$ \\
${ }_{14}^{40} \mathrm{Si}, 150 \mathrm{y}$ & 0 & 0.063 & 0.0027 & 0.002 & 0.068 & $0.13 / 0.092$ \\
${ }_{19}^{47} \mathrm{~K}, 1.3 \times 10^{9} \mathrm{y}$ & 0 & 0.48 & 0.022 & 0.04 & 0.54 & $1.82 / 1.75$ \\
${ }_{21}^{46} \mathrm{Cc}, 83.5 \mathrm{~d}$ & 0 & 0.12 & 0.0 & 0.01 & 0.13 & $0.026 / 0.036$ \\
${ }_{21}^{47} \mathrm{Sc}, 3.3 \mathrm{~d}$ & 0 & 1.05 & 0.024 & 0.12 & 1.19 & $3.13 / 4.09$ \\
${ }_{22}^{44} \mathrm{Ti}, 63 \mathrm{y}$ & 0 & 0.98 & 0.011 & 0.10 & 1.09 & $0.62 / 0.86$ \\
${ }_{23}^{50} \mathrm{~V}, 1.4 \times 10^{17} \mathrm{y}$ & 0 & 1.72 & 0.05 & 0.26 & 2.02 & $0.16 / 0.19$ \\
${ }_{24}^{51} \mathrm{Cr}, 27.7 \mathrm{~d}$ & 0 & 3.32 & 0.03 & 0.26 & 3.60 & $4.43 / 7.43$ \\
${ }_{25}^{54} \mathrm{Mn}, 312.3 \mathrm{~d}$ & 0 & 15.20 & 0.12 & 1.16 & 16.48 & $10.00 / 18.08$ \\
${ }_{26}^{55} \mathrm{Fe}, 2.7 \mathrm{y}$ & 0 & 11.68 & 0.08 & 0.55 & 12.31 & $14.32 / 30.00$ \\
${ }_{26}^{59} \mathrm{Fe}, 44.5 \mathrm{~d}$ & 0 & 53.66 & 0.25 & 2.43 & 56.33 & $19.32 / 42.79$ \\
${ }_{26}^{60} \mathrm{Fe}, 1.5 \times 10^{6} \mathrm{y}$ & 0.00 & 4.90 & 0.03 & 0.10 & 5.03 & $4.24 / 10.49$ \\
${ }_{27}^{56} \mathrm{Co}, 77.3 \mathrm{~d}$ & 0 & 9.71 & 0.08 & 0.54 & 10.32 & $0.80 / 1.98$ \\
${ }_{27}^{57} \mathrm{Co}, 271.8 \mathrm{~d}$ & 0 & 64.33 & 0.26 & 2.55 & 67.15 & $8.74 / 20.13$ \\
${ }_{27}^{58} \mathrm{Co}, 70.9 \mathrm{~d}$ & 0 & 55.52 & 0.17 & 1.57 & 57.26 & $32.44 / 77.45$ \\
${ }_{27}^{60} \mathrm{Co}, 5.3 \mathrm{y}$ & 0.02 & 63.12 & 0.24 & 1.25 & 64.63 & $56.61 / 138.06$ \\
${ }_{30}^{65} \mathrm{Zn}, 244.3 \mathrm{~d}$ & 0 & 1.80 & 0.02 & 0.22 & 2.04 & $26.28 / 66.12$ \\
& 0 & 0.04 & 0.18 & 8.77 & $19.58 / 62.78$ \\
\hline
\end{tabular}

TIVIA as well as the available experimental data from LUX [18] and Baudis et al. $[19,23]$.

The cosmogenic production rates from the Geant4-based simulations and the calculations of ACTIVIA are converted into radioactivity (decay rate) using the formula below:

$$
A(B q / k g)=\frac{\left(R \times\left(1-e^{\left(-\ln 2 \times T_{s} / t_{1 / 2}\right)}\right)\right) \times e^{\left(-\ln 2 \times T_{u} / t_{1 / 2}\right)}}{86400}
$$

where $R$ is the cosmogenic production rate in atoms per $\mathrm{kg}$ per day, $T_{s}$ is the exposure time on the surface, $t_{1 / 2}$ is the decay half-life, and $T_{u}$ is the decay 


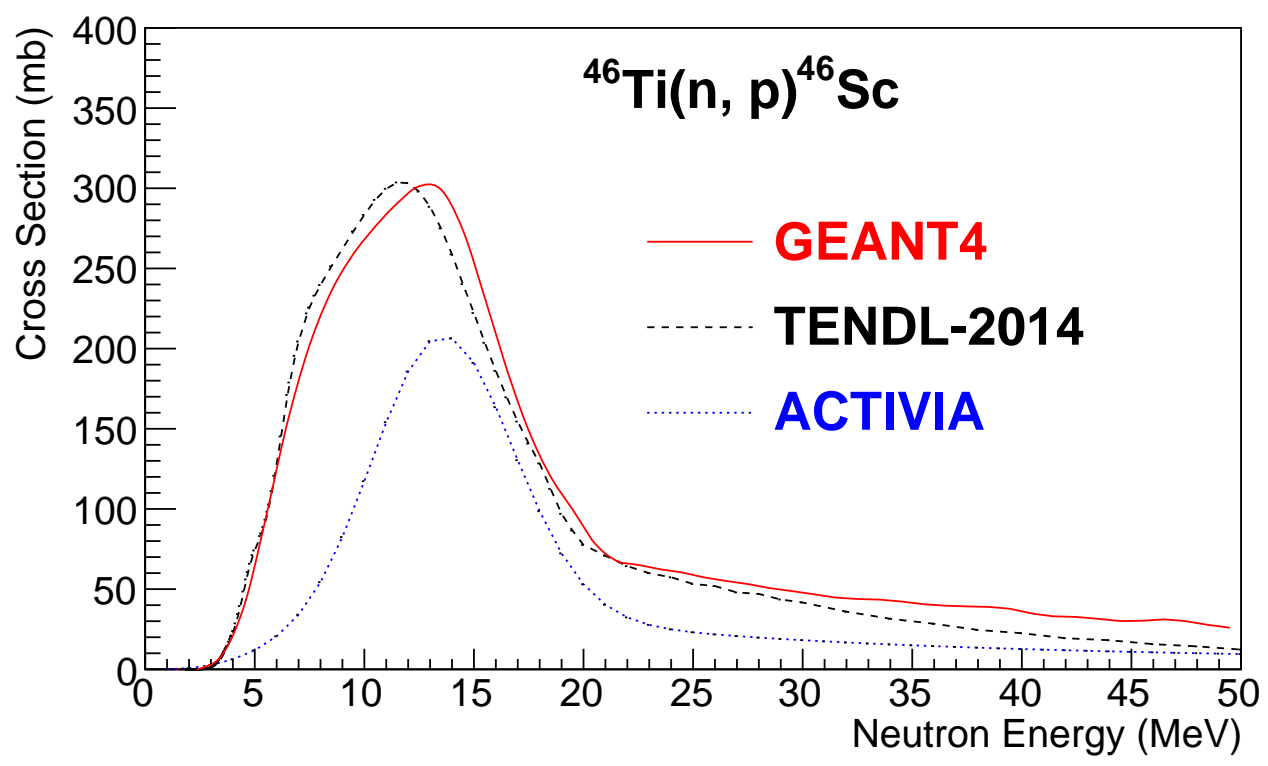

Fig. 7. Cross-sections comparison for the production of ${ }^{46} \mathrm{Sc}$ through ${ }^{46} \mathrm{Ti}(\mathrm{n}, \mathrm{p}){ }^{46} \mathrm{Sc}$ between Geant4, ACTIVIA, and TENDL-2014.

Table 4

Cosmogenic production in titanium at the sea level. Columns $2-5$ give Geant4 results.

\begin{tabular}{|l|llll|l||c|}
\hline Isotope,Half Life & $\mathrm{n}(<4 \mathrm{MeV})$ & $\begin{array}{c}\mathrm{n}(>4 \mathrm{MeV}) \\
\left(\mathrm{kg}^{-1} \mathrm{day}^{-1}\right)\end{array}$ & Muon & Proton & $\begin{array}{l}\text { Total } \\
\left(\mathrm{kg}^{-1} \mathrm{day}^{-1}\right)\end{array}$ & $\begin{array}{c}\text { ACTIVIA1 } / 2 \\
\left(\mathrm{~kg}^{-1} \mathrm{day}^{-1}\right)\end{array}$ \\
\hline${ }_{11}^{22} \mathrm{Na}, 2.6 \mathrm{y}$ & 0.00 & 0.20 & 0.00 & 0.08 & 0.28 & $0.93 / 0.79$ \\
${ }_{13}^{26} \mathrm{Al}, 7.2 \times 10^{5} \mathrm{y}$ & 0.00 & 0.41 & 0.02 & 0.10 & 0.52 & $1.02 / 1.02$ \\
${ }_{14}^{32} S i, 150 \mathrm{y}$ & 0.00 & 1.51 & 0.00 & 0.14 & 1.65 & $0.87 / 1.21$ \\
${ }_{19}^{40} \mathrm{~K}, 1.3 \times 10^{9} \mathrm{y}$ & 0.00 & 20.98 & 0.12 & 0.97 & 22.06 & $27.46 / 60.98$ \\
${ }_{20}^{47} \mathrm{Ca}, 4.5 \mathrm{~d}$ & 0.00 & 10.13 & 0.01 & 0.09 & 10.23 & $0.31 / 0.72$ \\
${ }_{21}^{46} S c, 83.8 \mathrm{~d}$ & 0.31 & 270.42 & 0.43 & 4.32 & 275.49 & $107.80 / 270.07$ \\
${ }_{21}^{47} S c, 3.3 \mathrm{~d}$ & 1.83 & 385.35 & 0.54 & 6.39 & 394.12 & $48.78 / 116.38$ \\
${ }_{22}^{44} \mathrm{Ti}, 63 \mathrm{y}$ & 0.00 & 95.94 & 0.46 & 3.68 & 100.08 & $4.84 / 12.03$ \\
${ }_{23}^{50} \mathrm{~V}, 1.4 \times 10^{7} \mathrm{y}$ & 0.00 & 0.32 & 0.01 & 0.07 & 0.39 & $4.40 / 14.13$ \\
${ }_{24}^{51} \mathrm{Cr}, 27.7 \mathrm{~d}$ & 0.00 & 0.02 & 0.00 & 0.00 & 0.02 & $0 / 0$ \\
\hline
\end{tabular}

time at the underground site before the measurement is taken. Note that the production rates in the simulations need to be corrected by altitudes where the experimental data were taken. The fluxes of muon and neutrons as a function of altitude is shown in Figure 2. 
Table 5

Cosmogenic production in stainless steel at the sea level. Columns 2 - 5 give Geant4 results.

\begin{tabular}{|c|c|c|c|c|c|c|}
\hline Isotope,Half Life & $\mathrm{n}(<4 \mathrm{MeV})$ & $\begin{array}{r}\mathrm{n}(>4 \mathrm{MeV} \\
\left(\mathrm{kg}^{-1} \mathrm{da}\right.\end{array}$ & $\begin{array}{l}\text { Muon } \\
\left.{ }^{1}\right)\end{array}$ & Proton & $\begin{array}{l}\text { Total } \\
\left(\mathrm{kg}^{-1} \mathrm{day}^{-1}\right)\end{array}$ & $\begin{array}{c}\text { ACTIVIA1/2 } \\
\left(\mathrm{kg}^{-1} \mathrm{day}^{-1}\right)\end{array}$ \\
\hline${ }_{4}^{7} B e, 53.1 \mathrm{~d}$ & 0.00 & 0.02 & 0.01 & 0.02 & 0.05 & $2.04 / 2.05$ \\
\hline${ }_{4}^{10} \mathrm{Be}, 1.5 \times 10^{6} \mathrm{y}$ & 0.00 & 0.10 & 0.00 & 0.01 & 0.11 & $0.93 / 0.89$ \\
\hline${ }_{6}^{14} C, 5.7 \times 10^{3} \mathrm{y}$ & 0.60 & 1.16 & 0.01 & 0.04 & 1.81 & $0.41 / 0.28$ \\
\hline${ }_{11}^{22} N a, 2.6 \mathrm{y}$ & 0.00 & 0.22 & 0.00 & 0.05 & 0.27 & $0.65 / 0.69$ \\
\hline${ }_{13}^{26} A l, 7.2 \times 10^{5} \mathrm{y}$ & 0.00 & 0.80 & 0.01 & 0.07 & 0.88 & $0.97 / 1.57$ \\
\hline${ }_{14}^{32} S i, 150 y$ & 0.00 & 0.32 & 0.02 & 0.05 & 0.39 & $0.31 / 0.30$ \\
\hline${ }_{19}^{40} \mathrm{~K}, 1.3 \times 10^{9} \mathrm{y}$ & 0.00 & 2.60 & 0.04 & 0.26 & 2.90 & $5.94 / 8.90$ \\
\hline${ }_{20}^{47} \mathrm{Ca}, 4.5 \mathrm{~d}$ & 0.00 & 0.97 & 0.00 & 0.03 & 1.00 & $0.18 / 0.42$ \\
\hline${ }_{21}^{46} S c, 83.8 \mathrm{~d}$ & 0.00 & 8.43 & 0.04 & 0.34 & 8.80 & $8.09 / 17.84$ \\
\hline${ }_{21}^{47} S c, 3.3 \mathrm{~d}$ & 0.00 & 8.77 & 0.02 & 0.29 & 9.08 & $3.54 / 8.14$ \\
\hline${ }_{22}^{44} T i, 63 \mathrm{y}$ & 0.00 & 12.14 & 0.15 & 0.98 & 13.27 & $0.86 / 1.69$ \\
\hline${ }_{23}^{50} \mathrm{~V}, 1.4 \times 10^{17} \mathrm{y}$ & 0.25 & 70.58 & 0.24 & 1.55 & 72.62 & $42.06 / 102.84$ \\
\hline${ }_{24}^{51} \mathrm{Cr}, 27.7 \mathrm{~d}$ & 3.94 & 282.47 & 5.34 & 6.86 & 298.61 & $88.92 / 222.34$ \\
\hline${ }_{25}^{54} M n, 312.3 \mathrm{~d}$ & 3.53 & 222.24 & 0.69 & 4.00 & 230.45 & $74.75 / 191.02$ \\
\hline${ }_{26}^{55} \mathrm{Fe}, 2.7 \mathrm{y}$ & 3.78 & 621.84 & 18.30 & 12.99 & 656.90 & $106.45 / 266.52$ \\
\hline${ }_{26}^{59} F e, 44.5 \mathrm{~d}$ & 0.14 & 0.31 & 0.00 & 0.01 & 0.45 & $0.08 / 0.20$ \\
\hline${ }_{26}^{60} \mathrm{Fe}, 1.5 \times 10^{6} \mathrm{y}$ & 0.00 & 0.08 & 0.00 & 0.00 & 0.08 & $0.02 / 0.05$ \\
\hline${ }_{27}^{56} C o, 77.3 \mathrm{~d}$ & 0.00 & 14.68 & 0.10 & 0.97 & 15.75 & $47.71 / 130.59$ \\
\hline${ }_{27}^{57} C o, 271.8 \mathrm{~d}$ & 0.00 & 79.12 & 0.15 & 1.47 & 80.74 & $14.74 / 36.07$ \\
\hline${ }_{27}^{58} C o, 70.9 \mathrm{~d}$ & 8.15 & 80.07 & 0.39 & 1.51 & 90.11 & $4.95 / 13.04$ \\
\hline${ }_{27}^{60} \mathrm{Co}, 5.3 \mathrm{y}$ & 0.00 & 6.17 & 0.01 & 0.10 & 6.27 & $1.81 / 4.92$ \\
\hline
\end{tabular}

\subsection{Comparison for the cosmogenic activity in natural xenon}

The LUX [43] experiment reported the measured activity of ${ }^{127} \mathrm{Xe}$ and ${ }^{133} \mathrm{Xe}$ utilizing their first three months of data $[18,43]$. In this work, an exposure time period of 150 days at sea level and appropriate exposures (7 to 49 days) at an altitude of 1480 meters are applied according to information recorded historically since the xenon gas bottles were produced [18]. Predicted and observed decay rates are listed in Table 6 after 90 days underground. 
Table 6

Decay rates of two xenon isotopes as calculated with Geant4 and ACTIVIA, and measured by LUX $[18,43]$ after 90 days cooling down underground.

\begin{tabular}{|l|l|c|c|c|}
\hline $\begin{array}{l}\text { Activated } \\
\text { Isotope }\end{array}$ & Target & $\begin{array}{c}\text { This Work } \\
(\mu \mathrm{Bq} / \mathrm{kg})\end{array}$ & $\begin{array}{c}\text { ACTIVIA1/2 } \\
(\mu \mathrm{Bq} / \mathrm{kg})\end{array}$ & $\begin{array}{c}\text { LUX data[18] } \\
(\mu \mathrm{Bq} / \mathrm{kg})\end{array}$ \\
\hline${ }^{127} \mathrm{Xe}$ & $\mathrm{Xe}$ & 470 & $73 / 180$ & $(490 \pm 95)$ \\
${ }^{133} \mathrm{Xe}$ & $\mathrm{Xe}$ & $7.0 \times 10^{-3}$ & $9.8 \times 10^{-4} / 2.4 \times 10^{-3}$ & $(25.0 \pm 5.0) \times 10^{-3}$ \\
\hline
\end{tabular}

The activation of xenon is also compared to the available data from Baudis et al. [19] as shown in Table 7 in where the saturation activity at sea level is used. Note that the saturation activity describes a saturation level at which its disintegration rate equals its production rate and allows a fair comparison between simulations/calculations and experimental data.

Table 7

Saturation activity at sea level assuming infinite exposure time.

\begin{tabular}{|l|l|c|c|c|}
\hline $\begin{array}{l}\text { Activated } \\
\text { Isotope }\end{array}$ & Target & $\begin{array}{c}\text { This Work } \\
(\mu \mathrm{Bq} / \mathrm{kg})\end{array}$ & $\begin{array}{c}\text { ACTIVIA1/2 } \\
(\mu \mathrm{Bq} / \mathrm{kg})\end{array}$ & $\begin{array}{c}\text { Baudis et al. data [19] } \\
(\mu \mathrm{Bq} / \mathrm{kg})\end{array}$ \\
\hline${ }^{113} \mathrm{Sn}$ & $\mathrm{Xe}$ & 67.6 & $45.7 / 61.3$ & $<55$ \\
${ }^{125} \mathrm{Sb}$ & $\mathrm{Xe}$ & 16.8 & $0.2 / 0.01$ & $590_{-230}^{+260}$ \\
${ }^{127} \mathrm{Xe}$ & $\mathrm{Xe}$ & 2670 & $413 / 1040$ & $1870_{-270}^{+290}$ \\
${ }^{133} \mathrm{Xe}$ & $\mathrm{Xe}$ & 1140 & $160 / 390$ & $<1200$ \\
\hline
\end{tabular}

It is clear that the results for ${ }^{127} \mathrm{Xe}$ and ${ }^{133} \mathrm{Xe}$ from the Geant4 simulation agree with the results from LUX and Baudis et al. reasonably well. However, the results from ACTIVIA are different from LUX and Baudis et al. by a factor of more than 2 depending on the neutron flux used in ACTIVIA. For example, for the production of ${ }^{127} \mathrm{Xe}$, the difference between ACTIVIA1 and the LUX data is a factor of 6.7 and the difference between ACTIVIA2 and the LUX data is a factor of 2.7 with ACTIVIA2 calculations that used more accurate neutron flux. This indicates a difference of a factor of 2.5 between ACTIVIA1 and 2 due to the difference in the assumed neutron flux. The difference of a factor of 2.7 between ACTIVIA2 and the LUX data may come from the cross section libraries used in the calculation of ACTIVIA2. We can conclude that the libraries for the cross sections of neutron inelastic scattering in Geant4, at least, for the production of ${ }^{127} \mathrm{Xe}$ and ${ }^{133} \mathrm{Xe}$ are adequate. 


\subsection{Comparison for the cosmogenic activity in natural titanium}

Cosmogenic activation of natural titanium can produce a radioactive isotope of ${ }^{46} \mathrm{Sc}$. We compare the results from the Geant4 simulation and ACTIVIA calculations to the LUX data [18] in Table 8, using an exposure time of 180 days for the titanium at 1480 meters altitude roughly equivalent to the LUX titanium history. As can be seen in Table 8, both Geant4 simulation and ACTVIA agree with the measured data reasonably well ( $<$ a factor of 2 difference). This indicates that the cross section libraries used in Geant 4 and ACTIVIA are adequate for the production of ${ }^{46} \mathrm{Sc}$.

Table 8

Saturation activity of ${ }^{46} \mathrm{Sc}$ from titanium activation at sea level.

\begin{tabular}{|l|l|c|c|c|}
\hline $\begin{array}{l}\text { Activated } \\
\text { Isotope }\end{array}$ & Target & $\begin{array}{c}\text { This Work } \\
(\mu \mathrm{Bq} / \mathrm{kg})\end{array}$ & $\begin{array}{c}\text { ACTIVIA1/2 } \\
(\mu \mathrm{Bq} / \mathrm{kg})\end{array}$ & $\begin{array}{c}\text { LUX data[18] } \\
(\mu \mathrm{Bq} / \mathrm{kg})\end{array}$ \\
\hline${ }^{46} \mathrm{Sc}$ & $\mathrm{Ti}$ & 7300 & $2900 / 7300$ & $(4400 \pm 300)$ \\
\hline
\end{tabular}

\subsection{Comparison for the cosmogenic activity in natural copper}

Cosmogenic activation of natural copper from a Geant4 simulation are compared to ACTIVIA, Cosmo [19], TALYS [20], and the available data [19] as shown in Table 9. The agreements between Geant4 and other calculations as well as the measurements from Baudis et al. are within a factor of 2 . One must point out that even though ACTIVIA1 with the original neutron spectrum (blue line in Fig. 1) shows better agreement with data than ACTIVIA2, this is not a valid agreement as discussed previously in section 2.2 and Figure 6 because the neutron spectrum used in ACTIVIA2 is more accurate based on the fit to the data.

\subsection{Tritium production}

${ }^{3} \mathrm{H}$, a long lived radioactive isotope ( $\beta$ decay), can be produced in all materials through muon spallation and neutron inelastic scattering. In Table 10 we tabulate the cosmogenic production rate of ${ }^{3} \mathrm{H}$ in various targets from the Geant4 simulation and other available calculations. It is worth mentioning that the production of ${ }^{3} \mathrm{H}$ in germanium and $\mathrm{NaI}$ targets from Geant4 is slightly higher than the earlier calculation using TALYS 1.0 [20]. A total of $42.87 \mathrm{~kg}^{-1} \mathrm{~d}^{-1}$ in $\mathrm{NaI}$ is consistent with a recent report of $\sim 40.6 \mathrm{~kg}^{-1} \mathrm{~d}^{-1}$ [44] from ANAIS-25 as shown in Table 10. Aside from silicon, the agreement is good between the Geant4 simulation and ACTIVIA2. 
Table 9

Saturation activity of different isotopes from activation of copper at sea level.

\begin{tabular}{|l|l|c|c|c|c|}
\hline $\begin{array}{l}\text { Activated } \\
\text { Isotope }\end{array}$ & $\begin{array}{l}\text { Geant4 } \\
(\mu \mathrm{Bq} / \mathrm{kg})\end{array}$ & $\begin{array}{c}\text { ACTIVIA1/2 } \\
(\mu \mathrm{Bq} / \mathrm{kg})\end{array}$ & $\begin{array}{c}\text { Cosmo } \\
(\mu \mathrm{Bq} / \mathrm{kg})\end{array}$ & $\begin{array}{c}\text { TALYS }[20] \\
(\mu \mathrm{Bq} / \mathrm{kg})\end{array}$ & $\begin{array}{c}\text { Baudis et al. data }[19] \\
(\mu \mathrm{Bq} / \mathrm{kg})\end{array}$ \\
\hline${ }^{46} \mathrm{Sc}$ & 12.4 & $36.2 / 47.4$ & 17.0 & - & $27_{-9}^{+11}$ \\
${ }^{54} \mathrm{Mn}$ & 136.0 & $165.7 / 347.2$ & 156 & 188 & $154_{-34}^{+35}$ \\
${ }^{59} \mathrm{Fe}$ & 99.5 & $49.1 / 121.5$ & 50 & - & $47_{-14}^{+16}$ \\
${ }^{56} \mathrm{Co}$ & 113.2 & $101.2 / 233$ & 81 & - & $108_{-16}^{+14}$ \\
${ }^{57} \mathrm{Co}$ & 747.7 & $375.5 / 896.4$ & 350 & 650 & $519_{-95}^{100}$ \\
${ }^{58} \mathrm{Co}$ & 644.4 & $655.2 / 1597.8$ & 632 & - & $798_{-58}^{+62}$ \\
${ }^{60} \mathrm{Co}$ & 713.2 & $295.7 / 744$ & 297 & 537 & $340_{-68}^{+82}$ \\
\hline
\end{tabular}

Table 10

The cosmogenic production rate of ${ }^{3} H$ at the sea level in various targets. Columns $2-5$ are obtained from the Geant 4 simulation.

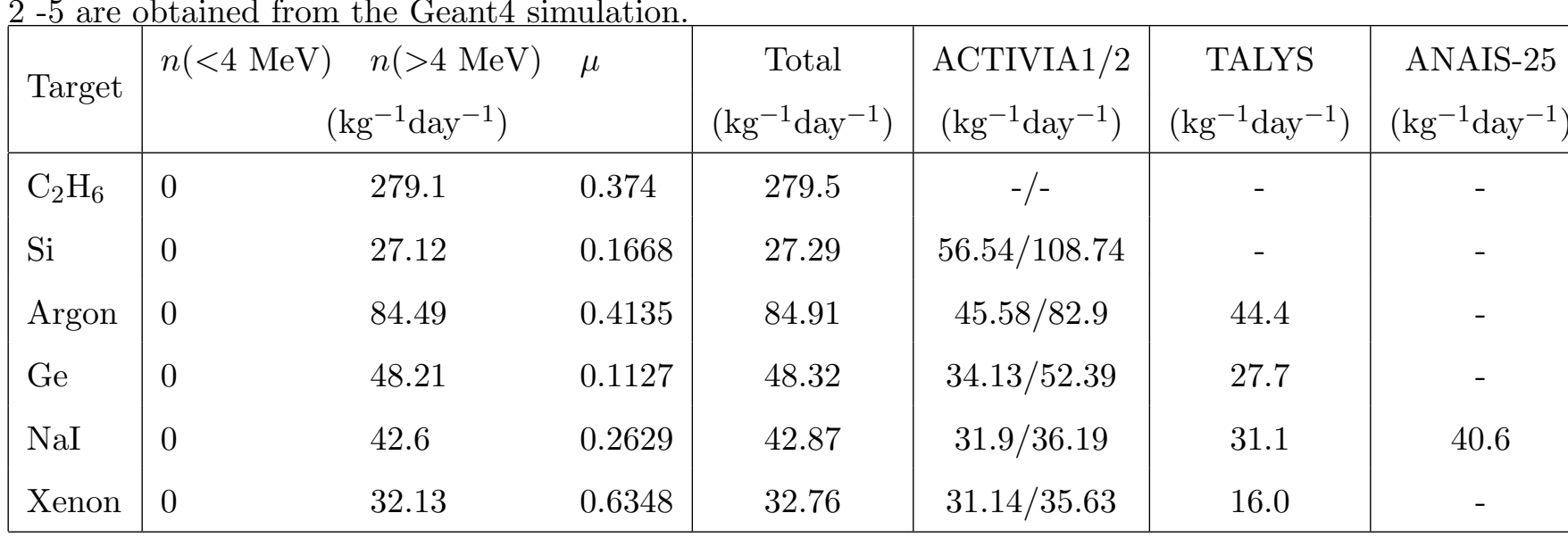

\section{Cosmogenic and radiogenic production underground}

Cosmogenic activation of materials in a underground environment can be estimated using the method described in Barker et al. [45]. Muon and muoninduced neutron fluxes are several orders of magnitude lower underground than at surface. The cosmogenic production underground is therefore often expected to be negligible. Since the radioactive isotopes in Tables 1-5 are mainly induced by fast neutrons from cosmic rays, and there are fast neutrons from natural radioactivity in any underground laboratory, we also evaluate radiogenic production for the most important isotopes relevant to xenon-based experiments. From the results of the simulations in Tables 1-5, the isotopes with higher production rates, longer half-life, and emission of either low energy $\mathrm{X}$-rays/Auger electrons $\left({ }^{127} \mathrm{Xe}\right)$ inside target or gamma rays with energies up to $\mathrm{MeV}\left({ }^{46} \mathrm{Sc}\right.$ and $\left.{ }^{60} \mathrm{Co}\right)$ in titanium or copper that are close to target, are 
problematic. Thus, the main concerns are the production of ${ }^{127} \mathrm{Xe}$ in xenon gas, ${ }^{46} \mathrm{Sc}$ in titanium, and ${ }^{60} \mathrm{Co}$ in copper and stainless steel. To understand the production rates underground, we use ${ }^{127} \mathrm{Xe}$ as an example. The production rate is 229 atoms $\mathrm{kg}^{-1} \mathrm{~d}^{-1}$ on the surface with a fast neutron flux (above 4 $\mathrm{MeV}$ ) of 0.004 neutrons $\mathrm{cm}^{-2} \mathrm{~s}^{-1}$. The capture process associated with thermal neutrons contributes 0.64 atoms $\mathrm{kg}^{-1} \mathrm{~d}^{-1}$ on the surface with a flux of 0.002 neutrons $\mathrm{cm}^{-2} \mathrm{~s}^{-1}$. We simulated the production rate for an underground laboratory where the fast neutron flux $\left(\mathrm{E}_{n}>4 \mathrm{MeV}\right)$ is about $10^{-6}$ neutrons $\mathrm{cm}^{-2} \mathrm{~s}^{-1}$ and the thermal neutron flux is about $10^{-7}$ neutrons $\mathrm{cm}^{-2} \mathrm{~s}^{-1}$ [46], using Geant4 to estimate the production rate of radioactive isotopes underground. The neutron energy spectrum from radiogenic processes obtained in Ref. [46], where U/Th contents and the density of rock are described, is used in the Geant4 simulation. We summarize the production rate of ${ }^{127} \mathrm{Xe}$ at SURF, $\sim 1480$ meters below the surface $[46,47]$. Note that the calculation is performed for xenon gas bottles without any shielding in the underground space.

- The possible maximum production rate from fast neutrons is less than $2.4 \times 10^{-3}$ atoms $\mathrm{kg}^{-1} \mathrm{~d}^{-1}$. The dominant activation rate is due to either $(\alpha$, $\mathrm{n})$ or spontaneous fission neutrons (depending on the rock composition) with neutron energies above $4 \mathrm{MeV}$, resulting from natural radioactivity [48].

- The possible production rate from thermal neutrons is about $7.5 \times 10^{-5}$ atoms $\mathrm{kg}^{-1} \mathrm{~d}^{-1}$.

- The possible production rate from cosmogenic activation through spallation is estimated to be $7.1 \times 10^{-4}$ atoms $\mathrm{kg}^{-1} \mathrm{~d}^{-1}$. For the neutrons from cosmicray muons, an approximate scaling method for neutron spectrum, which takes into account the average energy difference between the sea level and the underground, is used as described in Ref. [45]. The scaling factor is $\left(\frac{E_{\mu}^{u g}}{E_{\mu}^{s u}}\right)^{0.73} \times \frac{\Phi_{\mu}(u g)}{\Phi_{\mu}(s u)} \times R_{\text {iso }}(s u)$, where $\mathrm{E}_{\mu}^{u g}=321 \mathrm{GeV}$ is the average energy of muons at a depth of 1480 meters [45], $\mathrm{E}_{\mu}^{s u}=4 \mathrm{GeV}$ is the average energy of muons at the surface [45], $\Phi_{\mu}(u g)=4.4 \times 10^{-9} \mathrm{~cm}^{-2} \mathrm{~s}^{-1}$ is the total muon flux at a depth of 1480 meters, $\Phi_{\mu}(s u)=0.0347 \mathrm{~cm}^{-2} \mathrm{~s}^{-1}$ is the average muon flux weighted with the exposure dates at sea level and at the surface of SURF, $R_{\text {iso }}$ is the cosmogenic activation rate induced by neutrons at the surface.

The conclusion is that the production of ${ }^{127} \mathrm{Xe}$ underground is about 3 atoms per ton per day with a saturation activity of $3.5 \times 10^{-5} \mathrm{~Bq} /$ ton. This is a very low activity and only a tiny fraction of decays through emission of low energy X-rays/Auger electrons can cause background events for xenon-based dark matter experiments. Moreover, such sensitive detectors are protected by neutron shielding so during the science run the background rate due to activation is much smaller than from radioactivity in detector components. Activation induced before the target is shielded (during underground storage) will decay within a few months during commissioning phase of the experiment. 
We conclude that the radioactivity of these isotopes produced underground can be neglected.

\section{Conclusion}

The cosmogenic activations of several key components of the next generation rare event search experiments at sea level have been simulated using the GEANT4 and ACTIVIA packages. Fast neutrons, thermal neutrons and muons at the Earth surface are considered individually. The total production rates of several isotopes are compared with other calculations $[12,15,20]$ as well as the available experimental data from LUX [18] and Baudis et al. [19]. Significant differences are found in the results between Geant4 and ACTIVIA packages. We attribute these differences to the different input neutron energy spectra and the cross-section libraries used in the two packages. We emphasize the key requirement of using the accurate neutron energy spectrum and cross-sections for particular nuclear reactions. We have found that Geant4 can predict the production rates of different radioactive isotopes by cosmogenic activation pretty well.

\section{Acknowledgments}

The authors wish to thank Christina Keller and Wenzhao Wei for carefully reading of this manuscript. This work is supported in part by NSF PHY0758120, PHYS-0919278, PHYS-1242640, DOE grant DE-FG02-10ER46709, the Office of Research at the University of South Dakota and a 2010 research center support by the State of South Dakota. The simulations of this work was performed on High Performance Computing systems at the University of South Dakota. Electronic data exchange for this project was supported in part by NSF award ACI-1440681. V. A. Kudryavtsev contribution was supported by the Science and Technology Facilities Council (UK).

\section{References}

[1] G. R. Blumenthal, S. Faber, J. R. Primack and M. J. Rees, Nature 311, 517 (1984).

[2] M. Davis, G. Efstathiou, C. S. Frenk and S. D. M. White, Astrophys. J. 292, 371 (1985).

[3] C. L. Bennett, et al., ApJS., 208 (2013) $20 \mathrm{~B}$. 
[4] M. W. Goodman and E. Witten, Phys. Rev. D31, 3059 (1985).

[5] J. L. Feng, Ann. Rev. Astron. Astrophys. 48, 495 (2010).

[6] The LZ Collaboration, arXiv:1509.02910.

[7] The XENON Collaboration, arXiv:1507.07748.

[8] Steve R. Elliott and Petr Vogel, Annu. Rev. Nucl. Part. Sci. 115 (2002)

[9] Steven R. Elliott and Jonathan Engel, J. Phys. G: Nucl. Part. Phys. 30, R183 (2004).

[10] F.T Avignone III, G.S. King III and Yuri Zdesenko, New Journal of Physics (in press 2004).

[11] A.S. Barabash, Physics of Atomic Nuclei, 67, No. 3, 438 (2004).

[12] M. Agostini et al. (GERDA Collaboration), Phys. Rev. Lett 111 (2013) 122503.

[13] J. B. Albert et al. (The EXO-200 Collaboration), Nature 510, 229234 (12 June 2014).

[14] A. Gando et al. (KamLAND-Zen Collaboration), Phys. Rev. Lett. 110, (2013) 062502 .

[15] W. Xu et al., (Majorana Collaboration) Journal of Physics: Conference Series 606 (2015) 012004.

[16] A. Alfonso et al. (CUORE Collaboration), Phys. Rev. Lett. 115, 102502 (2015).

[17] R. Arnold et al. Eur. Phys. J. CDOI 10.1140/epjc/s10052-010-1481-5 (2010).

[18] D. S. Akerib et al. (The LUX Collaboration), Astropart. Phys. 62 (2015) 33-46, arXiv:1403.1299.

[19] Laura Baudis et al., arXiv: 1507.03792. EPJ C 75 (2015) 485.

[20] D.-M. Mei, Z.-B. Yin, and S. R. Elliott, Astropart. Phys. 31 (2009) 417-420, arXiv: 0903.2273.

[21] S. Agostinelli, et al., Nucl. Instr. and Meth. A506 (2003) 250-303; J. Allison et al, IEEE Transactions on Nuclear Science 53 (2006) 270-278.

[22] J. J. Back and Y. A. Ramachers, Nucl. Instr. and Meth. A586 (2008) 286-294.

[23] M. Laubenstein and G. Heusser, Appl. Rad. and Isot. 67 (2009) 750.

[24] http://www.slac.stanford.edu/comp/physics/geant4/slac_physics_lists/ shielding/physlistdoc.html.

[25] Thomas K. Gaisser, "Cosmic Rays and Particle Physics", Cambridge University Press 1990.

[26] J. R. Benbrook et al., Letter Al Nuovo Cimento, V3, N13, (1972) 557. 
[27] H. Bilokon et al., Journal of GeoPhysical Research, V94, (1989) 12145.

[28] M. Guan, et al., http://escholarship.org/uc/item/6jm8g76d.

[29] C. Hagmann, D. Lange, and D. Wright, UCRL-TM-229452, http://nuclear.llnl.gov/simulation/doc_cry_v1.7/cry_physics.pdf.

[30] M. S. Gordon, et al., IEEE Transactions on Nuclear Science 51, 3427 (2004).

[31] L. D. Hendrick and R. D. Edge, Phys. Rev. V145 (1966) 1023.

[32] J. F. Ziegler, IBM J. RES. DEVELOP. VOL. 40 NO. 1 JANUARY 1996.

[33] C. Hagmann, D. Lange, and D. Wright, 2007 IEEE Nuclear Science Symposium Conference Record V2 (2007) 1143-1146.

[34] Arjan Koning, "Status of TENDL: TENDL-2013 and 2014", May 2014, WPEC meeting, NEA Data Bank.

[35] R. Silberberg, C.H. Tsao, Astrophys. J. Suppl. 220 (I) 25 (1973) 315; R. Silberberg, C.H. Tsao, Astrophys. J. Suppl. 220 (II) 25 (1973) 335.

[36] R. Silberberg, C.H. Tsao, Astrophys. J. Suppl. 35 (1977) 129; R. Silberberg, C.H. Tsao, J.R. Letaw, Astrophys. J. Suppl. 58 (1985) 873; R. Silberberg, C. H. Tsao, J. R. Letaw, in: Proceedings of the 20th International Cosmic Ray Conference, Moscow, vol. 2, 1987, p. 133; R. Silberberg, C.H. Tsao, Phys. Rep. 191 (1990) 351; R. Silberberg, C.H. Tsao, A.F. Barghouty, Astrophys. J. 501 (1998) 911.

[37] F.K. McGowan, W.T. Milner, H.J. Kim, Wanda Hyatt, Nuclear Data Sheets. Section A Volume 7, Issues 12, Pages 1-232 (November 1969).

[38] A. Santamarina et al., The JEFF-3.1.1 Nuclear Data Library, Nuclear Energy Agency Organisation for Economic Co-operation and Development.

[39] K. Shibata, O. Iwamoto, T. Nakagawa, N. Iwamoto, A. Ichihara, S. Kunieda, S. Chiba, K. Furutaka, N. Otuka, T. Ohsawa, T. Murata, H. Matsunobu, A. Zukeran, S. Kamada, and J. Katakura: "JENDL-4.0: A New Library for Nuclear Science and Engineering," J. Nucl. Sci. Technol.. 48(1), 1-30 (2011).

[40] Yu.N. Shubin, V.P. Lunev, A.Yu. Konobeyev, A.I. Dityuk, International Nuclear Data Committee, INDC(CCP)-385, Distrib.:G.

[41] E. Aprile et al. (XENON100 Collaboration), Phys. Rev. Lett. 109 (2012) 181301.

[42] K. Abe et al. (The XMASS Collaboration), Phys. Lett. B724 (2013) 46-50.

[43] D. S. Akerib et al. (LUX Collaboration), Phys. Rev. Lett. 112 (2014) 091303.

[44] J. Amaré et al., AIP Conf. Proc. 1672, 140001 (2015); arXiv:1505.06102v1.

[45] D. Barker, D.-M. Mei, and C. Zhang, Phys.Rev. D86 (2012) 054001.

[46] D.-M. Mei et al., Astropart. Phys. 34 (2010) 33-39.

[47] Jaret Heise, arXiv:1503.01112, J.Phys.Conf.Ser. 606 (2015) 1, 012015.

[48] V. Tomasello et al. Astroparticle Phys. 34 (2010) 70. 